\title{
Plasmon Enhancement of Fluorescence in Single Light-Harvesting Complexes from Amphidinium carterae
}

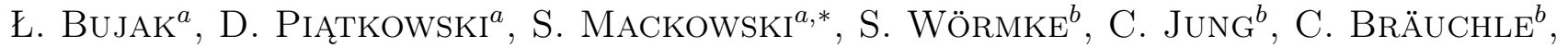 \\ A. Agarwal ${ }^{c}$, N.A. Kotov $^{c}$, T. Schulte $^{d}$, E. Hofmann $^{d}$, T.H.P. Brotosudarmo ${ }^{e}$ \\ H. Scheer ${ }^{e}$, A.O. Govorov ${ }^{f}$ AND R.G. Hiller ${ }^{g}$ \\ ${ }^{a}$ Institute of Physics, Nicolaus Copernicus University, Grudziądzka 5, 87-100 Toruń, Poland \\ ${ }^{b}$ Department of Chemistry and Biochemistry, Ludwig-Maximilian-University \\ Butenandtstrasse 11, D-81377 Munich, Germany \\ ${ }^{c}$ Department of Chemical Engineering, University of Michigan, Ann Arbor, Michigan 48109, USA \\ ${ }^{d}$ Department of Biology and Biotechnology, Ruhr-University Bochum, 44780 Bochum, Germany \\ ${ }^{e}$ Department of Biology I-Botany, Ludwig-Maximilian-University, D-80638 Munich, Germany \\ ${ }^{f}$ Department of Physics and Astronomy, Ohio University, Athens, Ohio 45701, USA \\ ${ }^{g}$ Department of Biological Sciences, Macquarie University, North Ryde, NSW 2109, Australia
}

\begin{abstract}
We show that single peridinin-chlorophyll $a$-protein light-harvesting complexes from dinoflagellate $A m p h i$ dinium carterae placed near to silver nanoparticles show strongly enhanced fluorescence emission. Single molecule spectroscopy experiments performed at room temperature point toward an enhancement of more than an order of magnitude for optimal conditions. Irrespective of the enhancement, we observe no effect of the metal nanoparticle on the fluorescence emission energy of the complex. This result provides a way to control the optical properties of biomolecules via plasmon excitations in metal nanoparticles.
\end{abstract}

PACS numbers: 87.80.Nj, 87.14.E-, 87.64.M-

\section{Introduction}

Plasmon resonances generated in metal nanoparticles have recently stimulated a considerable interest in various research fields such as optical spectroscopy, cell imaging and biosensor design [1-3]. This remarkable versatility stems from dramatic influence imposed by plasmons on the optical properties of dipoles located in their vicinity, as demonstrated for semiconductor nanocrystals $[4,5]$ and dye molecules [6]. It has been shown that the optical response of an emitter, characterized by radiative and non-radiative processes, coupled to a plasmonic structure depends upon the spatial arrangement as well as spectral characteristics of a studied system. The interplay between plasmon and the emitter influences both the radiative and non-radiative processes. It thereby determines whether the fluorescence of the emitter is enhanced or whether it is quenched due to the dominating role of non-radiative energy transfer from the dipole to the metal nanoparticle [6].

Metal-enhanced fluorescence (MEF) has been observed for many hybrid systems composed mainly of very sta-

* corresponding author; e-mail: mackowski@fizyka.umk.pl ble and highly fluorescing emitters, such as organic dyes and semiconductor nanocrystals and nanowires. In addition, attempts to influence the emission properties of weakly fluorescing systems such as DNA [7], carbon nanotubes [8], and light-harvesting complexes $[9,10]$ have been made. The light-harvesting complexes are protein-pigment systems containing chlorophyll (Chl) and carotenoid molecules embedded in a protein. They collect light energy and transfer it to reaction centers where the charge separation takes place, the first step in photosynthesis process.

In this work we study the effect of plasmon resonances on the optical properties of the peridinin-chlorophyll $a$-protein (PCP) complex from the dinoflagellate $\mathrm{Am}$ phidinium carterae. This water-soluble complex, whose structure has been determined by X-ray crystallography with $2 \AA$ resolution by Hofmann et al. [11], represents a unique example in the diverse class of light-harvesting antennas. While most of the antennas collect light energy predominantly by $\mathrm{Chl}$ or bacteriochlorophyll (BChl) molecules, the main pigment of PCP is a carotenoid, peridinin (Per), which absorbs light from 450 to $550 \mathrm{~nm}$, in a spectral region not available to the Chls. A single PCP monomer contains two Chl $a$ molecules and eight Per organized in two clusters (see inset in Fig. 1a). Each Per 
molecule is in van der Waals contact with the tetrapyrrole ring of the Chl $a$ of the same cluster. The center-to-center distance between the two Chls $a$ within a monomer is $17.4 \AA$. All the pigments are embedded in the hydrophobic cavity.

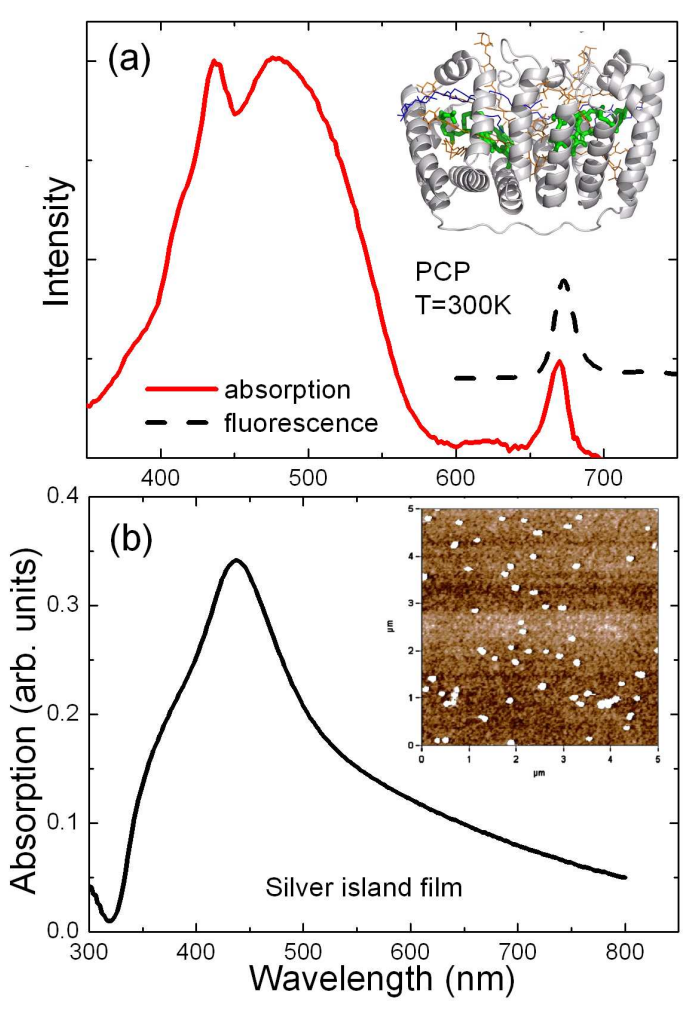

Fig. 1. (a) Absorption and fluorescence spectrum of a peridinin-chlorophyll $a$-protein complex. The excitation wavelength of the emission was $532 \mathrm{~nm}$. Inset: structure of a PCP monomer. (b) Absorption spectrum of the silver island film. Inset: AFM image of the silver islands.

Previous studies of PCP, carried out both on the ensemble $[12,13]$ and single-molecule level [14-16], have provided detailed understanding of the optical properties and their relation to the structure, as well as of the energy transfer processes between Per and Chls and between the Chls themselves. The absorption spectrum of PCP [15], displayed in Fig. 1a, shows that this complex utilizes Per as its primary pigment, which is responsible for the dominant absorption band in the blue-green spectral region (350 to $550 \mathrm{~nm}$ ). The Chls absorb around $668 \mathrm{~nm}\left(Q_{Y}\right.$ band) and $440 \mathrm{~nm}$ (Soret band). The fluorescence of $\mathrm{PCP}$, shown in Fig. 1a, originates from the $Q_{Y}$ transition of Chl $a$ and it is located at $673 \mathrm{~nm}$, more than $100 \mathrm{~nm}$ below the Per absorption band. The quantum efficiency of the energy transfer reaches up to $100 \%$ and involves an intramolecular charge transfer state [13]. In addition, circular dichroism experiments accompanied with theoretical calculations have suggested relatively weak dipole-dipole coupling (about $10 \mathrm{~cm}^{-1}$ ) between Chls within a single monomer [12]. This conclusion has been corroborated by recent single molecule experiments [15]. The spectral properties, in particular significant overlap between Per absorption and plasmon frequencies of metal nanoparticles, render PCP an ideal system for studying the impact of plasmonic interactions on the excitation and recombination dynamics in this relatively simple light-harvesting biomolecule.

\section{Materials and metods}

PCP complexes were obtained by biochemical reconstitution of the $N$-terminal domain apoprotein with Per and Chl $a$, as described in detail previously [14]. The final product, monomeric PCP reconstituted with Chl $a$, was equilibrated with TRIS buffer ( $5 \mathrm{mM}, \mathrm{pH} 7.6)$ and kept frozen until used.

Silver island films (SIFs) were prepared by reducing an aqueous silver nitrate solution [4]. All chemicals were purchased from Sigma-Aldrich and used as received. Briefly, freshly prepared aqueous $\mathrm{NaOH}$ (1.25 M) was added to a silver nitrate solution. The precipitate was re-dissolved by adding $\mathrm{NH}_{4} \mathrm{OH}$, and the solution was cooled to $\approx 5^{\circ} \mathrm{C}$ under stirring. After adding D-glucose, 12 clean microscope cover slips were dipped in the solution, which was then heated up to $30^{\circ} \mathrm{C}$. The resulting Ag-covered glass coverslips were examined using absorption spectroscopy and atomic force microscopy (AFM). The inset in Fig. 1b displays the AFM images of metal islands with diameters from $70-140 \mathrm{~nm}$ and heights between 30 and $40 \mathrm{~nm}$. As shown in Fig. 1b, the plasmon resonance has a maximum at $450 \mathrm{~nm}$ and the linewidth of about $150 \mathrm{~nm}$, and thus matches nicely the PCP absorption [10].

Silver nanowires were synthesized using the polyol process [17]. In a typical synthesis $3 \mathrm{ml}$ ethylene glycol solution of $0.1 \mathrm{M}$ silver nitrate and $3 \mathrm{ml}$ of ethylene glycol solution of $0.6 \mathrm{M}$ poly (vinyl pyrrolidone) (mol. wt. $55,000)$ were injected simultaneously into $5 \mathrm{ml}$ ethylene glycol refluxed at $160^{\circ} \mathrm{C}$ at a rate of $0.3 \mathrm{ml} \mathrm{min}^{-1}$. The reaction was continuously stirred using a magnetic stirrer and the reaction mixture was refluxed at $160^{\circ} \mathrm{C}$ for $60 \mathrm{~min}$. After cooling, the reaction mixture was diluted with acetone and centrifuged at $2000 \mathrm{rpm}$ for $20 \mathrm{~min}$ to separate nanowires from particles. The nanowires have an average diameter of $60 \pm 10 \mathrm{~nm}$.

Single molecule fluorescence measurements were performed at room temperature using a modified scanning confocal microscope (Zeiss LSM 410) equipped with a high numerical aperture (NA) oil-immersion objective (Zeiss Plan-Apochromat $63 \times / 1.4$ ). The samples were excited at $532 \mathrm{~nm}$. The emission was extracted using band pass filters (HQ 670/10, Chroma), dispersed by an Amici prism and detected by a Peltier-cooled CCD camera (Princeton Instruments, EEV 1300/100-EMB-chip) with exposure times of $1 \mathrm{~s}$. Desired concentrations of the light-harvesting complexes were achieved by diluting the PCP solution in 2\% aqueous PVA solution (Sigma-Aldrich). For the experiments on silver island films a 
$20 \mu \mathrm{l}$ drop of the sample was spin-coated on the SIF coverslips. Samples prepared on bare glass coverslips in exactly the same way were used as reference. The thickness of the PVA layer containing PCP complexes was about $100 \mathrm{~nm}$. For the experiments with Ag nanowires the solution of PCP was mixed together with the nanowires solution and then a $20 \mu \mathrm{l}$ drop of the sample was spin-coated on a coverslip.

\section{Experimental results}

In Fig. 2a we show the fluorescence spectra obtained for PCP complexes at ensemble concentration deposited on SIF substrates compared to the ones measured for standard glass coverslips. The complexes were excited at wavelength of $532 \mathrm{~nm}$, which corresponds largely to the absorption of Per. The data for the ensemble demonstrates clearly that the fluorescence emission of the light-harvesting complexes on the SIF is enhanced considerably, by a factor of 6 , as compared to the spectrum measured for the PCP on a glass substrate. We attribute this enhancement to the local increase of the electromagnetic field due to plasmons excited in metal islands. This observation is further corroborated by the spectra measured for individual PCP complexes, as shown in Fig. 2b. Here, representative fluorescence spectra obtained for single PCP complexes on both studied substrates are displayed. The average fluorescence enhancement observed for these complexes is, similarly to the ensemble data, sixfold. Importantly, analysis carried out for over a hundred of single PCP molecules reveals that the distribution of fluorescence intensities measured for $\mathrm{PCP}$ on glass substrate is much narrower than for these measured on SIF substrates. Indeed, in the first case the values range from 50 to 270 counts with $0.3 \mathrm{~s}$ acquisition time with an average value of 90. Remarkably, for PCP complexes deposited on SIF the fluorescence intensities vary from 60 to almost 2000 with the average value of 540 [10]. This result is similar to previously published results for CdTe nanocrystals on SIF [4]; it is a clear indication of inhomogeneities within the sample. Particularly important is the distribution of distances between a given PCP complex and a metal nanoparticle. From the preparation method it may range from essentially zero to $100 \mathrm{~nm}$, which is the thickness of the PVA layer. Since the distance between the dipole and the metal nanoparticle is one of the key parameters determining the effect of plasmons on the optical properties, such a broad distribution of fluorescence intensities is expected. Another factor contributing to the distribution of observed fluorescence intensities is the variation in size of the Ag nanoparticles. It is well known that the maximum of plasmon resonance, and, therefore, the electromagnetic field enhancement depends upon the size of the nanoparticle [18]. Nevertheless, the results of fluorescence spectroscopy obtained for PCP complexes on SIF substrate demonstrate unambiguously that plasmons in silver nanoparticles lead to huge, over an order of magnitude, enhancement of the fluorescence intensity.
This result opens a way to tune the optical properties of complex systems, such as light-harvesting antennae by means of plasmon engineering.

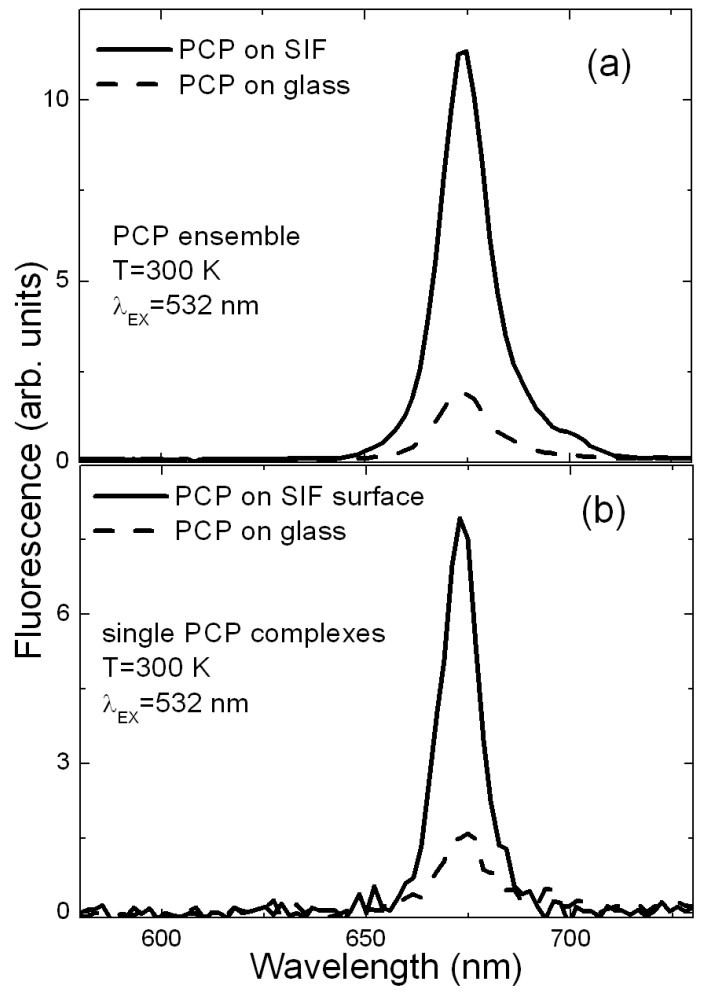

Fig. 2. (a) Fluorescence spectra measured for the PCP complexes at ensemble concentrations on SIF (solid curve) and on glass (dashed curve) substrates. (b) Representative fluorescence spectra measured for single PCP complexes on SIF (solid curve) and on glass (dashed curve) substrates. The excitation wavelength was $532 \mathrm{~nm}$, and the integration time was $0.3 \mathrm{~s}$ in all cases.

A natural step towards controlling the plasmon enhancement in biomolecules to a larger degree would be to study analogous effects for systems with metal nanoparticles of defined geometry. Such experiments should not only extend present understanding of the plasmon effect on the light-harvesting complexes, but can be important for developing theoretical models describing electromagnetic field enhancement in systems that contain many fluorophores in a defined geometry [5]. In Fig. 3 we show preliminary results obtained for a mixture of PCP complexes and Ag nanowires. The diameter of these nanowires is relatively well defined, with variations of less than $20 \%$, which is much smaller than the size distribution of nanoparticles fabricated in a SIF. The reflection image (Fig. 3a) shows the positions of the nanowires in the sample. Importantly, the concentration of the nanowires is low enough to observe every nanowire independently. The corresponding image displaying fluorescence of single PCP complexes is shown in Fig. $3 \mathrm{~b}$. Although PCP complexes are distributed on the cover- 


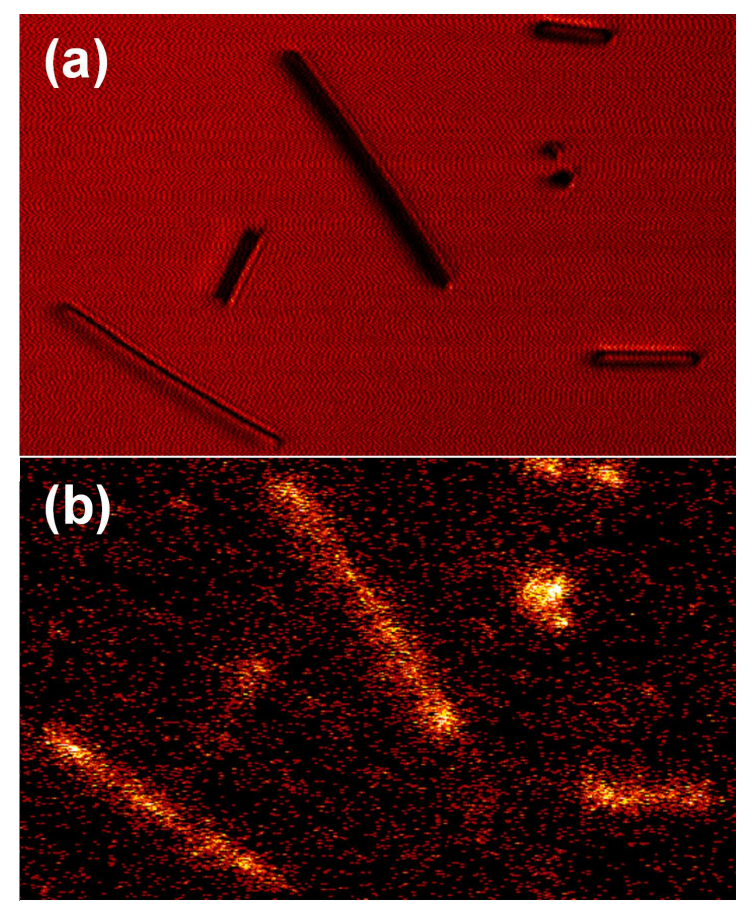

Fig. 3. Representative data obtained for PCP complexes mixed with Ag nanowires. (a) Transmission image of the nanowires. (b) Fluorescence image of the same sample area. Brightness of the spots corresponds to the intensity of the fluorescence emission. The excitation wavelength was $532 \mathrm{~nm}$. The horizontal size of the images is $30 \mu \mathrm{m}$.

slip approximately uniformly, the fluorescence originates predominantly from those located in the vicinity of the nanowires. Moreover, we observe a difference between PCP complexes located at the ends of the nanowires as compared to the ones located along the wire, indeed the fluorescence intensity of the former is approximately 3-to-4 times stronger than that of the latter. Future experiments will aim at a more quantitative analysis of these results, but it is already quite obvious that by tailoring parameters of metal nanostructures it is possible to control the plasmon interactions and therefore the strength of fluorescence enhancement.

\section{Conclusions}

Single molecule experiments demonstrate that the fluorescence of light-harvesting PCP complexes can be strongly enhanced when coupled to plasmon resonances excited in metal nanoparticles. The ability to tune the optical properties of complex molecules opens a way to design novel systems for efficient collection of sunlight for the purpose of energy conversion.

\section{Acknowledgments}

Financial support from the WELCOME program "Hybrid nanostructures as a stepping-stone towards efficient artificial photosynthesis" awarded by the Foundation for Polish Science is gratefully acknowledged.

\section{References}

[1] E. Ozbay, Science 311, 189 (2006).

[2] J. Lee, P. Hernandez, J. Lee, A.O. Govorov, N.A. Kotov, Nature Mater. 6, 291 (2007).

[3] J.R. Lakowicz, Plasmonics 1, 5 (2006).

[4] K. Ray, R. Badugu, J.R. Lakowicz, J. Am. Chem. Soc. 128, 8998 (2006).

[5] A.O. Govorov, G.W. Bryant, W. Zhang, T. Skeini, J. Lee, N.A. Kotov, J.M. Slocik, R.R. Naik, Nano Lett. 6, 984 (2006).

[6] P. Anger, P. Bharadwaj, L. Novotny, Phys. Rev. Lett. 96, 113002 (2006).

[7] J.R. Lakowicz, Y. Shen, S. D'Auria, J. Malicka, J. Fang, Z. Gryczynski, I. Gryczynski, Anal. Biochem. 301, 261 (2002).

[8] A. Hartschuh, H.N. Pedrosa, L. Novotny, T.D. Krauss, Science 301, 1354 (2003).

[9] M.H. Chowdhury, K. Ray, K. Aslan, J.R. Lakowicz, C.D. Geddes, J. Phys. Chem. C 111, 18856 (2007).

[10] S. Mackowski, S. Wörmke, A. Maier, T. Brotosudarmo, H. Harutyunyan, A. Hartschuh, A. Govorov, H. Scheer, C. Bräuchle, Nano Lett. 8, 558 (2008).

[11] E. Hofmann, P.M. Wrench, F.P. Sharples, R.G. Hiller, W. Welte, K. Diederichs, Science 272, 1788 (1996).

[12] F.J. Kleima, E. Hofmann, B. Gobets, I.H. van Stokkum, R. van Grondelle, K. Diederichs, van H. Amerongen, Biophys. J. 78, 344 (2000).

[13] D. Zigmantas, R.G. Hiller, V. Sundstrom, T. Polivka, Proc. Natl. Acad. Sci. USA 99, 16760 (2002).

[14] T.H.P. Brotosudarmo, E. Hofmann, R.G. Hiller, S. Wörmke, S. Mackowski, A. Zumbusch, C. Bräuchle, H. Scheer, FEBS Lett. 580, 5257 (2006).

[15] S. Wörmke, S. Mackowski, T.H.P. Brotosudarmo, C. Jung, A. Zumbusch, M. Ehrl, H. Scheer, E. Hofmann, R. Hiller, C. Bräuchle, Biochim. Biophys. Acta 1767, 956 (2007).

[16] S. Mackowski, S. Wörmke, T.H.P. Brotosudarmo, C. Jung, R.G. Hiller, H. Scheer, C. Bräuchle, Biophys. J. 93, 3249 (2007).

[17] Y.G. Sun, Y.N. Xia, Adv. Mat. 14, 833 (2002).

[18] S. Link, M. El-Sayed, J. Phys. Chem. B 103, 8410 (1999). 